\title{
Mais um ano que se encerra...
}

I ${ }^{1}$ Kenneth R. de Camargo Jr. I ${ }^{1}$ Instituto de Medicina Social, Universidade do Estado do Rio de Janeiro. Rio de Janeiro-RJ, Brasil (kenneth@uerj.br).
ORCID: 0000-0003-3606-5853

DOI: http://dx.doi.org/10.1590/S0103-73312018280400

O ano de 2018 marcou alguns aniversários significativos para a democracia e o Estado de Bem-Estar Social: setenta anos da Declaração Universal dos Direitos Humanos e do início do National Health Service do Reino Unido, e quarenta anos da conferência de Alma-Ata. Mas também trouxe à lembrança tempos tristes, marcando cinquenta anos da edição do AI-5 e o endurecimento da ditadura no Brasil, bem como a violenta repressão à Primavera de Praga.

A chegada de 2019, por sua vez, encontra o mundo numa certa encruzilhada política. Os resultados de referendos ou eleições recentes parecem sinalizar certa fadiga do status quo na política, dando a vitória a candidatos ou ideias que se apresentaram como outsiders, mesmo que não o fossem. Os eventos citados no primeiro parágrafo também marcaram à sua época rupturas importantes com a politics as usual, embora nem sempre na direção de maior liberdade, direitos ou segurança. Em que direção nos levará a história nos meses e anos à frente?

O triunfo global da ideologia neoliberal trouxe, nos seus rastros, crises frequentes por si só desestabilizadoras não só da economia como da política. Na observação arguta do jornalista Georges Monbiot:

[t]alvez o impacto mais perigoso do neoliberalismo não seja a crise econômica que causou, mas a crise política. [...] [N]a grande democracia do consumidor ou do acionista, os votos não são distribuídos igualmente. $\mathrm{O}$ resultado é uma falta de poder dos pobres e da classe média. Como os partidos da direita e da esquerda adotam políticas neoliberais 
semelhantes, a falta de poder se transforma em privação de direitos. Chris Hedges observa que "os movimentos fascistas constroem sua base não dos politicamente ativos, mas dos politicamente inativos, os 'perdedores' que sentem, muitas vezes corretamente, que náo têm voz ou papel a desempenhar no establishment político”. (MONBIOT, 2016).

A corrosão da confiança se estende, com ou sem razão, não apenas à classe política, mas a tudo aquilo que é percebido como "elite": a mídia tradicional e mesmo a ciência em geral são engolfadas pela crise de credibilidade. Não foi à toa que a organização Oxford Dictionaries declarou, à mesma época do comentário de Monbiot, "post-truth" (pós-verdade) como a palavra do ano de 2016.

Essa conjunção de desesperança e cinismo que estilhaça a própria ideia de verdade, ainda que contingente, traz ressonâncias desconfortáveis com um momento ainda mais dramático na História. Benjamin Hett, na sua magistral análise da derrocada da República de Weimar, sintetiza essa dimensão da seguinte forma: “

[a] hostilidade à realidade traduziu-se em desprezo pela política, ou melhor, desejo por uma política que, de alguma forma, não era política: algo impossível de existir. O funcionamento da democracia visto de perto - os acordos necessários, favores, compromissos - raramente são inspiradores. [...] Para que uma democracia funcione, todas as partes têm que reconhecer que têm pelo menos algum ponto em comum e que os compromissos são possíveis e necessários. Na década de 1930, no entanto, restava muito pouco desse espírito, à medida que a sociedade alemã se tornava cada vez mais amargamente dividida. Defensores da República muitas vezes pareciam pouco mais do que defensores de um sistema corrupto. Os opositores da democracia, pregando uma "antipolítica" de unidade e ressurreição, poderiam parecer que estavam operando em uma base moral mais elevada. (HETT, 2018, índice 49-50/698)

Face a tais tendências presentes em todo o mundo, inclusive em nosso meio, cabe a todos nós, pesquisadores, professores, profissionais e estudantes da Saúde Coletiva, defender os ideais democráticos, solidários e inclusivos que caracterizam nosso campo. Em particular e especificamente, a defesa do financiamento do SUS, da pesquisa e da universidade pública e gratuita como instituiçôes que asseguram a formulação e implementação de políticas coerentes com esses ideais.

Com o encerramento de mais um ano, é o momento de reconhecermos mais uma vez o trabalho essencial dos nossos revisores, sem o qual nenhum periódico científico existiria. Destacamos como revisor do ano o professor Carlos Alberto Ribeiro Costa, do Departamento de Psicologia da Universidade Federal Fluminense, campeão de 
2018 no número de pareceres; homenageamos por seu intermédio todas as pessoas que colaboraram com a Physis, que têm seus nomes listados em ordem alfabética ao final deste editorial.

Que tenhamos todos um feliz 2019, e que a chama da ciência e da Saúde Coletiva continue sempre a brilhar em nosso país.

\section{Referências}

HETT, B. C. The Death of Democracy. New York: Henry Holt \& Co., 2018 (online).

MONBIOT. G. Neoliberalism - the ideology at the root of all our problems. The Guardian, edição online de 15 abr. 2016. Disponível em: <https://www.theguardian.com/books/2016/ apr/15/neoliberalism-ideology-problem-george-monbiot>. Acesso em: dez 2018.

ADRIANA CASTRO

AGLEILDES QUEIRÓS

ALAN SILVA

ALDEN NEVES

ALESSANDRA MENDES

ALEXANDRA PENA

ALEXANDRE COSTA LEAL

ALEXANDRE COSTA-VAL

ALEXANDRE PALMA

ALICE MENEZES

ALTAMIRA PEREIRA

ANA CLÁUDIA FIGUEIRÓ

ANA LUIZA VILLASBÔAS

ANA PAULA HEMMI

ANDRÉ FENNER

ANDRÉ LUIZ DA SILVA

ANTONIO ROSSELLO

BÁRBARA FONSECA C.C. ANDRADA

BIANCHA ANGELUCCI

CARLA PEREIRA

CARLOS ANDRÉ ARRUDA
CARLOS ALBERTO R. COSTA

CARLOS GONÇALVES SERRA

CARLOS GUILHERME DO VALLE

CARLOS MOREIRA

CARLOS PAIXÃO JUNIOR

CAROLINA NOGUEIRA

CESAR AUGUSTO FAVORETO

CHARLES DALCANALE TESSER

CIRLENE CHRISTO

CLAUDIA BOCCA

CLAUDIA HENSCHEL DE LIMA

DANIELA AMADOR

DANIELLE BORDIN

DEBORAH DE AZEVEDO

DEIDVID ABREU

DENISE PIMENTA

DIEGO ANDRADE

EDINALDO CARMO

EDUARDO MELO

EDUARDO WERMELINGER

EFIGÊNIA FERREIRA 
ELAINE MIRANDA

ELISANGELA COSTA

ELISETE CASOTTI

ELIZABETHE CRISTINA SOUZA

ENRIC NOVELLA

EUGENIE NERI

FABIANA BOLELA

FABIANA KRAEMER

FABIOLA STOLF BRZOZOWSKI

FERNANDA ALZUGUIR

FERNANDA FERNANDES

FERNANDA LOPES

FERNANDA MONTES

FLAVIA CAMPOS

FLAVIA RAMOS

FRANCISCO INÁCIO BASTOS

GABRIELE CARVALHO

GISELE O'DWYER

GRACIELA SEHNEM

HENRIQUE NARDI

ILVANA GOMES

ISABELA SANTOS

ISABELLA MARTINS

ISSA DAMOUS

JAIME NEIRA

JOÃO SCATENA

JORGE MACHADO MESQUITA

JOSÉ ANTONIO VÁSQUEZ-MEDINA

JOSÉ JUNGES

JOSÉ MARÇAL JACKSON FILHO

JOSÉ RICARDO AYRES

JOSÉ RIVALDO FRANÇA

JOSENAIDE ENGRACIA DOS SANTOS

JUAREZ FURTADO
JULIANA CASEMIRO

JÚLIO ASSIS SIMÓES

KALLINE DE SOUZA

KALLINE FLÁVIA SILVA DE LIRA

KAREN ATHIÉ

KELLEM VINCHA

KHALED ALMAHNOUD

LEILA SENNA MAIA

LENIR SILVA

LEONARDO SAVASSI

LEONILDO SILVA

LÍGIA MARIA VIEIRA-DA-SILVA

LILIAN KOIFMAN

LILIAN MIRANDA

LUCAS MELO

LUCIANA SIMAS

LUCIENE JIMENEZ

LUCIENE ROCINHOLI

LUCILA NASCIMENTO

LUIZ FERNANDO ROJO

LUIZ TEIXEIRA

LUIZA CAZOLA

LUIZA COSTA

LUIZA MARIA CUNHA

LUNA FREITAS SILVA

MAGDA CHAGAS

MAGDA RIBEIRO DE CASTRO

MAGDA SCHERER

MÁNCEL MARTÍNEZ

MARCELA LÓPEZ

MARCIA AGOSTINI

MARCOS ANDREI

MARCOS NASCIMENTO

MARCOS VIRMOND 
MARCUS TEIXEIRA

MARI LUZ ESTEBAN

MARÍA INÉS BRINGIOTTI

MARIA LUCIA BOARINI

MARIANE PANSERA

MARILENA D. V. CORREA

MARINA NUCCI

MARIO JORGE SOBREIRA

MARTINHO BRAGA BATISTA E SILVA

MOÊMIA MIRANDA

MONICA DANTAS

NATÀLIA MAICAS CARCELLER

NEIDE EMY KUROKAWA E SILVA

NEUSA COLLET

NILIA PRADO

NINA SOALHEIRO PRATA

NURIA MALAJOVICH

PABLO DI LEO

PATRICIA BRAGA

PATRÍCIA CAVALCANTI

PAULA GAUDENZI

PAULA LAND

PAULO HENRIQUE ALMEIDA

RODRIGUES

PILAR BELMONTE

PRISCILA ALVES

PRISCILA ARAGÃO

RAFAEL LIMA

RAPHAELLA DAROS

REGINA FLAUZINO

REGINALDO MENDONÇA

RENATA MONTEIRO
RICARDO PENA

RITA HELENA FERREIRA GOMES

ROBERTA OLIVEIRA

RODRIGO MONTEIRO

ROGERIO AZIZE

RONALDO TEODORO DOS SANTOS

ROSANA ONOCKO CAMPOS

ROSÂNGELA GUERRINO

ROSENI PINHEIRO

ROSSANO CABRAL LIMA

SANDRA CAPONI

SELMA FRANCO

SHIRLEY DONIZETE PRADO

SIMONE MONTEIRO

SIMONE PAULON

STELLA TAQUETTE

SUELY MARINHO

SUZANA CANEZ

TERESA TONINI

TÚLIO BATISTA FRANCO

VANESSA MAIA RANGEL

VANIRA PESSOA

VARLEY DIAS SOUSA

VERONICA ALCOFORADO

VERUSKA ALEXANDRE

VILMA DIUANA

VIRGINIA MOREIRA

WILLIAM WAISSMANN

WILLIAN SANTOS

XAVIER MEDINA

XIMENA BERMUDEZ

YOLANDA BODOQUE 